\title{
Prevalence of neuropathic component of pain in a cohort of patients admitted to an Internal Medicine Department for chronic pain
}

\author{
Giuseppe Chesi, ${ }^{1}$ Giovanni Scanelli, ${ }^{2}$ Fabio Gilioli, ${ }^{3}$ Erio Scalabrini, ${ }^{1}$ Claudio Giumelli, ${ }^{1}$ Francesca Franco ${ }^{1}$ \\ ${ }^{1}$ Internal Medicine Unit, C. Magati Hospital, Scandiano (RE); ${ }^{2}$ Internal Medicine Unit, Arcispedale Sant'Anna, Ferrara; \\ ${ }^{3}$ Internal Medicine Unit, B. Ramazzini Hospital, Carpi (MO), Italy
}

\begin{abstract}
Due to the increasing age of the population the number of people suffering from chronic pain has significantly increased. People with chronic pain suffer from various diseases. Often this pain is not adequately controlled and is refractory, while its neuropathic component, which requires a different treatment, is perhaps underestimated compared to more properly nociceptive pain. The purpose of this study was to evaluate the presence of a neuropathic component in a cohort of 105 patients consecutively admitted to three Internal Medicine Units in Emilia Romagna. For the identification of the component of neuropathic pain diagnostic (DN4) questionnaire, previously validated, has been used. The average age of the patients studied was 64.4 years. The group of subjects with chronic non-cancer pain (78\%) was numerically higher than the group of patients suffering from cancer pain (22\%). All patients had pain and, according to the visual analogue scale (VAS), pain ranged from moderate to severe (median 7). Although without reaching statistically significant data, according to the VAS scale, cancer pain had an average higher value than non-cancer pain (7 vs 6.5). The prevalence of neuropathic component of pain was higher in patients with non-cancer pain $(66 \% v s 57 \%)$. Instead, the recorded pain intensity in patients with neuropathic component was statistically much higher than the group in which the neuropathic component was absent $(6.9$ vs $6.1 ; \mathrm{P}<0.05)$. In patients suffering from chronic pain, regardless of its nature and its etiology, the presence of a neuropathic component is significant. We must become aware of it and must search for it regularly through appropriate tools, such as the DN4 questionnaire. The presence of a neuropathic component usually makes the pain more intense and more refractory to treatments commonly used. Search for it may have therapeutic implications, suggesting that doctors use drugs active on this component. Since the majority of patients suffering from chronic pain are admitted to internal medicine wards, this awareness ought to become cultural heritage for the internist.
\end{abstract}

\section{Introduction}

With the progressive ageing of the population and the increase of chronic diseases, chronic pain has become common in non-cancer patients.

Meanwhile the awareness of this problem is increas-

Correspondence: Giuseppe Chesi, viale Martiri della Libertà 2, 42019 Scandiano (RE), Italy.

Tel.: +39.0522.850447 - Fax: +39.0522.850501.

E-mail: chesig@ausl.re.it

Key words: chronic pain, neuropathic pain, neuropathic pain diagnostic questionnaire.

Received for publication: 24 October 2013.

Revision received: 18 April 2014.

Accepted for publication: 21 April 2014.

This work is licensed under a Creative Commons Attribution NonCommercial 3.0 License (CC BY-NC 3.0).

CCopyright G. Chesi et al., 2015

Licensee PAGEPress, Italy

Italian Journal of Medicine 2015; 9:57-60

doi:10.4081/itjm.2015.441 ing among patients and health care professionals. In the past few years legislators have intervened several times to facilitate the access to the best health care for all patients affected by this problem, as pointed out in Law 38 recently approved by the Italian Parliament. ${ }^{1}$

Legislative measures have made it easier for physicians to prescribe opiates and made pain assessment during hospitalization mandatory.

However optimal pain control is not always achieved due to the presence of a neuropathic component of pain as well as to a nociceptive component. The treatment of the neuropathic component ${ }^{2}$ requires its recognition and, at the same time, the use of drugs different from those usually employed in nociceptive pain.

A simple and specific score for the detection of this component of pain is the neuropathic pain diagnostic (DN4) questionnaire ${ }^{3}$ which has been chosen due to its simplicity, facility of administering and reproducibility according to scientific validate literature. The aim of this study was to evaluate a series of patients affected by chronic pain admitted to three internal medicine wards of Emilia-Romagna. Using the DN4 questionnaire the prevalence of the neuropathic component of pain was determined in a cohort of patients. 


\section{Materials and Methods}

The study consisted in all patients being consecutively admitted to three Internal Medicine Units in Emilia Romagna [Scandiano (RE), Carpi (MO) and Ferrara Hospitals] between May and September 2012 with a diagnosis of chronic pain disorder. Pain intensity was recorded according to the visual analogue scale (VAS) scale in all patients. The DN4 questionnaire was then delivered (Figure 1).

All patients who had a DN4 score $\geq 4$ were considered affected by the neuropathic component of pain as reported by Bouhassira et al. with a sensitivity of 82 , $9 \%$ and a specificity of $89,9 \% .^{3,4}$

The care providers were allowed to choose their own pain treatment and the study had not been thought to test

\section{DN4 Questionnaire}

Please complete this questionnaire by ticking one answer for each item in the 4 questions below:

\section{INTERVIEW OF THE PATIENT}

Question 1: Does the pain have one or more of the following characteristics?

$$
\begin{aligned}
& \text { 1-Burning } \\
& \text { 2-Painful cold } \\
& \text { 3-Electric shocks }
\end{aligned}
$$

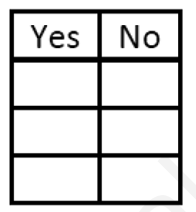

Question 2: Is the pain associated with one of more of the following symptoms in the same area?

$$
\begin{aligned}
& \text { 4- Tingling } \\
& 5-\text { Pins and needles } \\
& 6-\text { Numbness } \\
& 7-\text { Itching }
\end{aligned}
$$

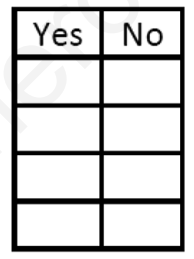

\section{EXAMINATION OF THE PATIENT}

Question 3: Is the pain located in an area where the physical examination may reveal one or more of the following characteristics?

8 - Hypoesthesia to touch

9 - Hypoesthesia to prick

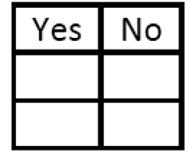

Question 4: In the painful area, can the pain be caused or increased by:

$10-$ Brushing

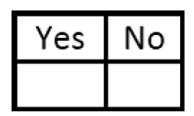

The total score is calculated as the sum of the 10 items and the cut-off value for the diagnosis of neuropathic pain is a total score of $4 / 10$.

Total

Figure 1. Neuropathic pain diagnostic (DN4) score for the evaluation of the neuropathic component of pain. Modified from Bouhassira et al., 2005. ${ }^{4}$ 
the efficacy of the drugs on the different components of pain. The most frequently used drugs for this purpose in the units involved were opiates (oxycodone, transdermal fentanyl and various preparations of slow-release morphine) and, at a lower extent, non-steroidal anti-inflammatory drugs (NSAIDs), gabapentin or pregabalin, carbamazepine and tricyclic antidepressant as also reported by more recent indications of the Literature. ${ }^{5}$

\section{Results}

A total of 105 patients (61 females and 44 males) were enrolled in the study. The average age was 64.4 years (+/-15.2 to $26-90$; median 67 years). The majority of patients $(78 \%)$ were affected by non-cancer pain, versus $22 \%$ by cancer pain. The average level of pain showed significantly high values with a median of 7 points. The comparison between cancer and non-cancer pain showed no statistically significant differences, although cancer patients showed a higher average pain trend $(7.0+/-1.9 v s 6.5+/-1.8)$. The administration of the DN4 questionnaires using as cut-off the value of $\geq 4$ showed the presence of neuropathic pain in 67 patients $(63 \%)$. The prevalence of pain with a neuropathic component was not significantly different between the group of patients with cancer and the other with no cancer. In the overall population study the average value obtained with the test DN4 was 3.8+/-1.4. Dividing the patients into two groups, oncological and non-oncological, a statistically significant difference in the prevalence of neuropathic component of pain was not achieved (57 vs 66\%; $\mathrm{P}=0.26$ ). However, when using the VAS scale to compare the intensity of pain in the group of patients with a neuropathic component versus the group of patients without a neuropathic component, a statistically significant difference $(6.9+/-1.7$ vs $6.1+/-$ $1.9 ; \mathrm{P}<0.05$ ) was found (Table 1).

\section{Discussion}

Neuropathic pain is usually present in some situations and, more specifically, in neurological diseases such as the phantom limb syndrome, diabetic neuropathy, neuralgia (especially post-viral) and in patients with sequelae of spinal cord damage. ${ }^{6,7}$ Even in Oncology the perception of the presence of a neuropathic component in this context is growing. ${ }^{8,9}$ Neuropathic pain as a component of chronic pain in general is rarely considered, and little evidence is found in the scientific literature. ${ }^{10}$ Some studies performed by Spanish general practitioners showed that neuropathic pain may also be present in a significant percentage of subjects seen for chronic pain in outpatient clinics. ${ }^{11}$ The peculiarity of our study was to evaluate patients affected by chronic pain in internal medicine wards. In these wards the percentage of patients suffering from cancer chronic pain is not all-encompassing. Furthermore, hospitalized patients may be regarded as selected for more serious diseases and with a greater impact of pain. Even in general wards these symptoms are not easy to control, especially when particularly intense. ${ }^{12}$ As reported by the literature, such difficulty in controlling the symptoms is probably not only due to real refractoriness, but also to inadequate treatment that very often neglects the neuropathic component in the choice of pharmacological options. ${ }^{13}$

Table 1. Summary of the results obtained with the subdivision of the cases among the individual participating centers.

\begin{tabular}{lcccc}
\hline Center & Total & Carpi & Ferrara & Scandiano \\
\hline Numbers & 105 & 32 & 21 & 52 \\
\hline Average age & $64.4(26-90)$ & $60.4(26-82)$ & $68.2(48-85)$ & $69.1(43-90)$ \\
\hline Male & $49(46.7 \%)$ & $14(43.7 \%)$ & $9(42.8 \%)$ & $26(50 \%)$ \\
\hline Female & $56(53.3 \%)$ & $18(56.3 \%)$ & $12(57.2 \%)$ & $26(50 \%)$ \\
\hline Non cancer pain & $82(78 \%)$ & $15(46.9 \%)$ & $19(90.5 \%)$ & $48(92.3 \%)$ \\
\hline Cancer pain & $23(22 \%)$ & $17(53.1 \%)$ & $2(9.5 \%)$ & $4(7.7 \%)$ \\
\hline VAS median & $6.6+/-1.8$ & $6.8+/-1.8$ & $4.9+/-1.2$ & $7.2+/-1.0$ \\
\hline Non cancer pain VAS median & $6.5+/-1.8$ & - & $4.8+/-1.3$ & $7.1+/-1.1$ \\
\hline Cancer pain VAS median & $7+/-1.9$ & $6.9+/-2.0$ & - & - \\
\hline Point DN4 score & $3.8+/-1.4$ & $3.6+/-1.1$ & - & - \\
\hline DN4 score $\geq 4$ & $67(63 \%)$ & $17(53.2 \%)$ & $10(47.6 \%)$ & $39(75 \%)$ \\
\hline Non cancer pain DN4 score $\geq 4$ & $54(66 \%)$ & $7(46.7 \%)$ & $10(52.6 \%)$ & $37(77 \%)$ \\
\hline Cancer pain DN4 score $\geq 4$ & $13(57 \%)$ & $11(64.1 \%)$ & 0 & $2(50 \%)$ \\
\hline
\end{tabular}

VAS, visual analogue score; DN4, neuropathic pain diagnostic. 
In our study, the proportion of patients with noncancer pain was significantly higher than the proportion of patients with cancer pain. In the first group, despite the lower pain intensity, the percentage of neuropathic pain component was higher than in the group of cancer patients. It should be emphasized how the pain reported by patients with the neuropathic component was significantly higher than the pain reported by those without this component. These data seem to confirm that subjects suffering from chronic pain in which there is also a neuropathic component present on average a greater intensity of pain. ${ }^{14}$ Our study did not take in consideration differences in individual treatments and therefore, whether this greater severity of pain is to be considered as an independent fact or it is rather, as suggested by others, ${ }^{13}$ due to an incomplete and not entirely correct treatment of the neuropathic component, ${ }^{15}$ remains controversial. Other studies showed that a neuropathic component of pain may be present making it more difficult to achieve pain control, for example an important neuropathic component was shown in a cohort of patients apparently affected only by osteoarticular pain. ${ }^{9}$

In the light of our contribution it is important that internists and also the nursing staff working in internal medicine wards learn to recognize this component of chronic pain and use appropriate scores (for example DN4) to confirm clinical suspicion. ${ }^{16}$ Nowadays such awareness is of great importance to set a more comprehensive therapeutic regimen including also drugs active on the neuropathic component of pain, avoiding the indiscriminate dosage increase of others medications (opiates, NSAID, etc.), non-optimal pain relief and frequent and serious side effects..$^{17,18}$

A specific analysis regarding modifications in the use of analgesic treatment as a result of the increased attention in the neuropathic component of chronic pain through the DN4 questionnaire systematic administration has not been carried out. The department's physicians have definitely an increased perception of the need to expand the range of therapeutic interventions against chronic pain, as well as increased attention to implement therapeutic interventions specifically oriented to treat its different components. Future studies are currently being planned about modification of analgesic treatment as a result of the increased attention to pain, its measurement and recognition of its various components.

\section{Conclusions}

In the past few years the scientific community has showed great interest in the different components of chronic pain and particularly in the neuropathic component. This component appears to be quite common not only in the area of neurological diseases or cancer, but also in general practice and internal medicine. It is there- fore mandatory that all doctors working in general or internist wards become able to use the most appropriate tools for the identification of neuropathic pain with the aim of reducing the number of patients with falsely refractory chronic pain and making the best possible use of available drugs for this insidious component of pain.

\section{References}

1. Ministero della Salute. Legge 15 marzo 2010, n. 38. Disposizioni per garantire l'accesso alle cure palliative e alla terapia del dolore. GU n. 65, 19/03/2010.

2. Kerstman E, Ahn S, Battu S, et al. Neuropathic pain. Handb Clin Neurol 2013;110:175-8.

3. Bouhassiraa B, Attala N, Fermanianc J, et al. Development and validation of the Neuropathic Pain Symptom Inventory. Pain 2004;108:248-57.

4. Bouhassira B, Attala N, Alchaar H, et al. Comparison of pain syndromes associated with nervous or somatic lesions and development of a new neuropathic pain diagnostic questionnaire (DN4). Pain 2005;114:29-36.

5. Kalso E, Aldington DJ, Moore RA. Drugs for neuropathic pain. BMJ 2013;347:f7339.

6. Baron R, Binder A, Wasner G. Neuropathic pain: diagnosis, pathophysiological mechanisms, and treatment. Lancet Neurol 2010;9:807-19.

7. Spaic M, Petkovic S, Tadic R. Electrophysiological evaluation of chronic neuropathic pain of spinal cord injury origin. Indian J Neurotrauma 2010;7:47-54.

8. Urch CE, Dickenson AH. Neuropathic pain in cancer. Eur J Cancer 2008;44:1091-6.

9. Bennett MI, Rayment C, Hjermstad M, et al. Prevalence and aetiology of neuropathic pain in cancer patients: a systematic review. Pain 2012;153:359-65.

10. Hochman JR, Gagliese L, Davis AM, Hawker GA. Neuropathic pain symptoms in a community knee OA cohort. Osteoarthritis Cartilage 2011;19:647-54.

11. Blanco E, Galvez R, Zamorano E, et al. Prevalencia del dolor neuropático (DN), según DN4, en atención primaria. Semergen 2012;38:203-10.

12. Jongen JL, Hans G, Benzon HT, et al. Neuropathic pain and pharmacological treatment. Pain Pract 2013;10:1-13.

13. Torrance N, Ferguson JA, Afolab E, et al. Neuropathic pain in the community: more under-treated than refractory? Pain 2013;154:690-9.

14. Smith BH, Torrance N. Epidemiology of neuropathic pain and its impact on quality of life. Curr Pain Headache Rep 2012;16:191-8.

15. Dworkin RH, O'Connor AB, Backonja M, et al. Pharmacologic management of neuropathic pain: Evidencebased recommendations. Pain 2007;132:237-51.

16. Bouhassira D, Attal N. Diagnosis and assessment of neuropathic pain: the saga of clinical tools. Pain 2011;152: S74-83.

17. Bjorkman DJ. Current status of nonsteroidal anti-inflammatory drug (NSAID) use in the United States: risk factors and frequency of complications. Am J Med 1999; 107:3S-10.

18. de Leon-Casasola OA. Opioids for chronic pain: new evidence, new strategies, safe prescribing. Am J Med 2013;126:S3-11. 